\title{
Processing Trade-Offs in Non-Native Learners' Performance of Narrative Tasks
}

\section{Mohamed Ridha Ben Maad}

Exploring learners' processes of memory and analysis has captivated considerable attention among language-learning researchers due to the recent prevalence of key concepts from feeder disciplines such as cognitive psychology and phraseology. However, there has been little empirical effort to describe the nature of interaction between these two processing modes. This article reports on a study that was designed (a) to explore the distribution of these two modes of language representation in the oral production of non-native learners of English and (b) to determine whether they shift their processing styles (i.e., lexical retrieval to rule analysis or vice versa) in the face of increasing cognitive load. Thirty Tunisian undergraduate students of English performed three narrative tasks over three tape-recorded episodes. Analysis of the transcribed findings revealed that these participants activated their memory-based system for lexical retrieval at the beginning of their performance when the tasks were not demanding and fell back on the rule-based mode when faced with the increasing processing load due to time pressure. These results empirically validate the role of formulaicity in second/foreign-language learners' processing styles.

L'étude des processus de mémoire et d'analyse chez les apprenants a beaucoup attiré l'attention des chercheurs en apprentissage de langue en raison de l'apport récent de disciplines connexes telles la psychologie cognitive et la phraséologie. Toutefois, on a fait peu d'effort empirique pour décrire la nature de l'interaction entre ces deux modes de traitement. Cet article porte sur une étude conçue pour (a) examiner la distribution de ces deux modes de représentation langagière dans la production orale d'apprenants en ALS et (b) déterminer si les apprenants changent de styles de traitement (c'est à dire de l'extraction lexicale à l'analyse de règles et inversement) face à une augmentation de la charge cognitive. Trente étudiants tunisiens aux études de premier cycle en anglais ont accompli trois tâches narratives au cours de trois séances enregistrées. L'analyse des résultats transcrits a révélé que les participants activaient leur système reposant sur la mémoire pour l'extraction lexicale au début de la tâche quand elle n'était pas exigeante, et avaient recours au mode basé sur les règles quand les contraintes temporelles augmentaient la charge de traitement. Ces résultats valident de façon empirique le rôle de la formulaicité dans les styles de traitement des apprenants de langue seconde ou étrangère. 


\section{Background}

The limited-capacity concept garnered considerable attention in Skehan's (1998) task-based framework. This framework focuses on how tasks lead language-learners selectively to allocate their attention across three performance areas: fluency, complexity, and accuracy. A trade-off relationship between the three performance areas arises from the level of difficulty stemming from a given task because each of the areas has its own function (i.e., fluency for communication, complexity for restructuring, and accuracy for grammar). According to Skehan, in order for L2 learners to balance their speaking abilities, they should: (a) increase the degree of lexicalization to streamline realtime processing (i.e., fluency), (b) work toward a more lexically and syntactically elaborate interlanguage system (i.e., complexity), and (c) gain more control over the rule-based system (i.e., accuracy).

Although the model has been widely adopted as an assessment tool in L2 literature, full agreement has not been reached in the language-learning literature as to whether attention trade-offs occur between, say, accuracy and complexity (Foster \& Skehan, 1996) or between fluency and accuracy (Yuan \& Ellis, 2003). The issue stems from, among other things, ${ }^{1}$ defining the nature of these performance windows in accordance with their corresponding processing components. In this respect, Wolfe-Quintero, Inagaki, and Kim (1998), drawing on the basic information-processing concepts of automaticity and restructuring, maintain that fluency-focused processing is driven by automaticity whereas restructuring is the driving force behind complexity-focused processing.

The competition between the performance areas stands as a surface manifestation of two processing modes: an analytic processing mode and a holistic processing mode. The analytic processing mode operates in the purview of second-language (L2) learners' analytic ability, which is, according to Skehan (1998), "the capacity to infer rules of language and make linguistic generalizations and extrapolations" (p. 207). More precisely, this capacity represents learners' grammatical sensitivity to attend to the formal accuracy and appropriateness in either language input or output, as well as the inductive ability to abstract rules and patterns from language samples both implicitly or explicitly. As a matter of knowledge representation, Skehan believes that analytical ability, or analyzability, represents a rule-governed system that is "parsimoniously and elegantly organized, with rules being compactly structured" (p. 88). Skehan concurs in Widdowson's (1989) reference to analyzability as system as a resource and not simply as an abstract system of parameters and configurations in the Chomskyan sense. The functioning of this mode of representation, Skehan posits, is cognitively demanding because "rules need complex processes of construction to underpin their operation, in which the units from which they are composed are necessarily small, and require detailed attention" (p. 89). 
The holistic processing mode functions on the basis of a lexically based memory system. The accessibility to or retrieval of lexical items of varied sizes is holistic, in the form of whole units that are not subject to internal manipulation. These lexical items are formulaic sequences holistically internalized and remembered as multi-word chunks bearing an idiomatic dimension (Pawley \& Syder, 1983; Weinert, 1995; Widdowson, 1989). Formulaic sequences are organized sequentially in real-time production, and they appear in the forms of collocations, clauses, and long phrasal stretches and even holophrases. It is worth mentioning here that the ensemble of formulaic sequences, regardless of their morphosyntactic variations, account for a large part of normal language use. Sinclair (1991), in this respect, claims that language production is controlled by an "idiom principle" determined by our "natural tendency to economy of effort; or it may be motivated in part by the exigencies of real-time conversation" (p. 110).

When engaged in cognitively taxing tasks, speakers typically resort to the holistic processing mode because of its processing advantage over the analytic processing mode. This mode, also called the exemplar-based system, is described by Skehan (1998) as a system that "lacks parsimony, and has only a limited generative potential" because its lexical constituents "do not require excessive internal computation, since they can function as integrated wholes" (p. 89). In fact, it is misleading to believe that we are totally creative in the Chomskyan sense of language use because this might overburden our capacity to attend to output. Skehan's account of the examplar-based mode is theoretically embedded in the master concept of communicative competence. It dovetails with Bachman's (1990) categorization, with the three component competences (linguistic, discourse, and sociolinguistic) being mediated by a pervasive strategic competence. Therefore, this attention-free processing mode has the advantage of maintaining fluent communication through the holistic retrieval of lexical units.

One issue worth addressing in regard to these two processing modes is the nature of the relationship between them: whether they are complementary to each other or interact competitively. In this respect, Skehan, like Pawley and Syder (1983), Weinert (1995), and Widdowson (1989), holds that both modes balance each other in the form of a formulaic-creative continuum. Being independent of each other does not deny the synergy that their interaction brings to the oral product. The assumption that speech is largely formulaic still needs a minimal degree of analyticity, where according to Widdowson, rules "are not generative but regulative and subservient" (p. 135). Nevertheless, this view lacks some clarity because it seems that these researchers consistently focus on describing a mature native speaker's (NS) dual-mode system rather than a fledgling non-native speaker (NNS) model, where the former has more automated rules and greater formulaic repertoire. In other words, because of the ca- 
pacity-related differences between NSs and NNSs, it would appear simplistic to assert that they share the same type of interaction between their processing modes.

Temple (2000) is among the few studies that address the difference between French NNS and NS production models with regard to the limitedcapacity concept. She noticed that NSs, unlike NNS who experienced clear hesitation and frequent pauses in their speech in difficult tasks, skillfully alternated between the two processing modes with no detrimental effect on their fluency. In the same vein, Foster (2001), in a reexamination of findings reported in Foster and Skehan (1996), observed that under cognitively demanding conditions, her NS informants resorted to formulaic language, and when pressure eased they switched to the analytic mode to produce more syntactically complex language. Foster explained that much of the formulaic content used "was not propositional but functioned as discourse organizers and fillers" (p. 89). With familiar tasks and planned tasks, NSs' preference for analytic language is explained by their meaning-biased pursuit of originality, and its exigencies cannot be satisfied by the conventionalized and idiomatic word combinations.

Both Foster (2001) and Temple (2000) found a reverse processing engagement pattern with regard to their NNS informants. Under high task pressure, the informants scored poorly in terms of lexicalized language use. However, when they performed under less taxing task conditions, the proportion of formulaicity in their output increased to the benefit of fluency. Overall, it follows that switching between the two processing modes is capacity-driven, and so the trade-off for a rule-based approach (i.e., through frequent pauses and repairs as indicators of controlled processing) is solely attributable to the limitation of the NNS examplar-based system. Interestingly enough, these findings question the widely accepted belief of Skehan (1998) and his co-researchers that decisions about allocating attention are simply determined by task difficulty. Instead, the availability of formulaic language in one's interlanguage may enable a more incisive account of the interaction patterns between the two processing modes and their subsequent effect on the surface areas of oral performance.

Prompted by the theoretical significance of the analysis advanced by Foster (2001), this study aims to draw empirical attention to formulaicity and its significance for on-line language processing. In this respect, the following hypotheses are suggested.

- NNSs draw on (a) the holistic-processing mode under low time pressure, and (b) the analytic-processing mode under high time pressure.

- The availability of formulaic language control trade-offs between the areas of fluency, complexity, and accuracy. 


\section{Method}

\section{Participants}

Thirty undergraduate students of English ( 25 women, 5 men) at the University of Tunis participated in this study. The participants, aged between 18 and 23, had comparable scores on an English-language proficiency test, and they were estimated to be at a low-intermediate level. As native speakers of Tunisian Arabic, they had all studied English for an average of 6.7 years in a classroom environment. Two assistants to the field worker took part in the coding procedure of this study. The first was a NNS teacher well experienced in language-testing, and the second was a visiting teacher from the United States with limited experience in applied linguistics. Both teacher and student participants were informed of the purpose of the research, and the latters' anonymity was preserved. The field worker made it clear that the students' participation would have no bearing on their class-related results.

\section{Procedure}

The informants were asked to perform three narrative tasks (Fletcher, 1997) based on sequenced picture prompts in a watch-and-tell style. Their performances were tape-recorded, transcribed in standard orthography, and coded for subsequent analysis. The tasks were administered during out-ofclass sessions spread over five months. This time span seemed appropriate for performing the tasks regularly each month at equal intervals. During this experimental period, the participants performed narrative tasks over six episodes, and only three of the six tasks were tape-recorded for subsequent transcribing, coding, and analysis (90 transcripts). All the tasks were sequenced similarly; each participant was given 15 minutes to plan his or her performance without any guidance from the field worker. Focus on three of six tasks for recording was judged reasonable to obtain as many data as necessary for analysis given the exacting nature of the transcribing and coding procedures.

The content of each task seemed to be of an optimal length to ensure a time-pressure effect during task engagement. The tasks were based on one discourse mode where the performers described events based on pictures in a watch-and-tell style. The participants were to serialize data sequentially as all the participants had similar designs: that is, 11 picture prompts in total for every story. Therefore, these tasks differed only in terms of topic. For example, the first task was about a rock band biography whereas the last involved a story of a lottery jackpot winner. The format of these tasks was a modified version of an activity series from a teaching resource book, Picture Stories Please (Fletcher, 1997) designed for intermediate EFL learners. In fact, with their cognitive load, the three tasks shared a "schematic sequential structure," which is based on a cause-effect pattern (Tavakoli \& Skehan, 2005). This structural format is less cognitively demanding than that of un- 
structured narratives, which comprise loosely related or disconnected events that might lead to disruptions in communication. During administration of the task, the field worker did not discuss speaker participants' performances with them in an effort to avoid any external effect (i.e., a form-focused or meaning-focused feedback) on their output or any potential type of bias.

In the study we sought to explore variations in L2 production as a function of switching between the two processing modes according to the degree of cognitive pressure. In the design, time pressure was chosen as the withinsubjects independent variable to measure task difficulty. According to Skehan (1998), time pressure stands for the communicative stress that reflects the "urgency with which a task needs to be completed" (p. 100). The operationalization of the time variable proceeded into two levels: the first 60 seconds (Time 1 ) and the last 60 seconds (Time 2 ) of each narrative performance. Time 1 was presumed to mark pressure-free and well planned oral production (i.e., 15 minutes were allotted to plan the tasks), whereas Time 2 was believed to underline the mounting difficulty occasioned by time pressure. ${ }^{2}$ Also, it is worth noting that the decision to limit the analyzed time intervals to one minute was prompted by (a) the relatively short performance ranges (i.e., 3-4 minutes) and (b) the huge workload of transcription and coding with respect to both discourse analytic and formulaicity measures.

As far as the dependent variables are concerned, both discourse analytical measures of fluency, complexity, and accuracy and measures of formulaicity were implemented. The performance areas of fluency, complexity, and accuracy were respectively represented by (a) Speech rate/Second (number of syllables divided by 60), Number of Pauses (1 pause $>0.5$ second), and Reformulations; (b) Subordinate clauses/T-unit; and (c) Errors/T-unit. These measures were chosen for analysis because of their construct validity and wide use in output-focused research. The formulaicity variable was operationalized into three dimensions of formulaic sequences (FLS): frequency of use, size, and proportion in the narrative output of the informants, corresponding to the measures of Number of FLSs/Min., Words per FLS, and Percentage of FLS (i.e., the proportion of formulaicity in one's spoken output). The use of these three measures was motivated by the need to capture more patterns of variation in the use of FLS in the two time intervals. FLS measures were claimed to attest to holistic-processing mode, whereas the attention-calling measures of Pauses and Reformulations were to identify the analytic-processing mode.

The most strenuous research task in this study was the coding procedure. The 90 transcripts were first coded according to the discourse analytic measures and then according to FLS units. Before undertaking the task of locating and counting FLSs, I held three meetings with my assistants to inform them about the identification and classification procedures to be adopted, for example, focus on formal criteria of non-compositionality and fixedness (Read \& Nation, 2004) to identify formulaic sequences that are either fixed or partly 
compositional. In the identification procedure, illustrations from Foster's (2001) review of formulaic categories were referred to for judging the formulaicity of any lexical sequence.

At first, I compared my count with that of my assistants (i.e., $20 \%$ of the transcripts) and found a substantial extent of disagreement. As a result, following the technique used by Foster (2001), the three judges worked together to reach consensus over problematic FLS instances and a convergence of tally. To satisfy the internal reliability assumption, $10 \%$ of the established results were further compared against a tally of the same sample that I made two months later, thus yielding high agreement percentages (e.g., Number of FLSs / minute=92.33).

Once the data were computer-coded and processed by SPSS application, both descriptive and inferential statistics were applied to evaluate the hypotheses that prompted this study. However, before reporting the analysis of the findings, a validation procedure was undertaken to examine whether the normality and homogeneity assumptions underlying data distribution were satisfied. As to sample normality assumption, it was satisfied across all the formulaicity and discourse analytic measures, because the data across these measures were not heavily skewed as none of the measures exceeded the absolute skewness value of 2.00/-2.00 (Tabachnick \& Fidell, 2001). Equally satisfied was the homogeneity assumption across all the six dependent variables, as illustrated by the nonsignificant results of the Levene's Test of Homogeneity of Variance ranging between (Errors/T-unit) $p=.069$ and $p=.995$ (Speech Rate/Sec.). Therefore, subsequent correlational and univariate and multivariate tests could be validly interpreted.

\section{Results}

In the study I aimed to capture how both holistic and analytic processing modes operate in relation with time pressure. In order to define the nature of interaction between these two processing modes, a Pearson correlation analysis was performed on formulaicity data (i.e., indicating holistic processing) and pausing and reformulation data (i.e., signifying analytic processing). Results reported in Table 1 demonstrate that all the correlation coefficients are significant at a 0.01 level. Positive correlations are particularly located between the measures representative of either processing mode, as with pauses and reformulations $(r=.59)$. However, a pattern of negative correlations is manifest between measures belonging to varying processing modes. For example, the analytic processing measure of Pauses demonstrates substantial but negative correlation with the three holistic processing measures of formulaicity (i.e., Number of FLSs [ $r=-.61]$; Words per FLS [ $r=-.36]$; Percent of FLS [ $r=-5] 7)$. These results confirm that the relationship between the two processing modes is marked by conspicuous competition where gains in one processing mode are mirrored by reductions in the other processing mode. 
Table 1

Correlational Results for Analytic and Holistic Measures

\begin{tabular}{lccccc}
\hline Variable & 1 & 2 & 3 & 4 & 5 \\
\hline 1. FLS/Min. & - & $.33^{*}$ & $.79^{\star}$ & $-.61^{*}$ & $-.64^{*}$ \\
2. Words/FLS & & - & $.45^{\star}$ & $-.36^{\star}$ & $-.28^{\star}$ \\
3. FLS \% & & & - & $-.57^{*}$ & $-.55^{\star}$ \\
4. Pauses & & & - & $.59^{\star}$ \\
5. Reformulations & & & & - \\
\hline
\end{tabular}

* Correlation is significant at the 0.01 level.

If the correlation results reveal the nature of relationship between the two processing modes, they provide no patterns of attentional focus on either mode. Such patterns can be distinguished through the distribution of formulaicity data across the two levels of the time pressure variable. Table 2 illustrates formulaicity through three dimensions: frequency of use, lexical density of formulaic units, and proportion of use in total performance. In fact, the mean distribution of formulaicity under the three measures is marked by a substantial reduction from the level at Time 1, an indicator of high time pressure. That is, the more the speakers advanced in the task of narrating, the less they drew on their exemplar-based depository. Note that the difference margins across the three measures are almost invariable although the rate of FLS frequency in Task $1(M=20.00)$ appears to be comparatively lower than in the other tasks ( $M=23.07$ for Task 2; $M=23.53$ for Task 3$)$. The least instance of variation documented is at the level of formulaic density, meaning that the dwindling size of the formulaic units used by the speakers was not proportionate with the reduction in frequency of use.

Table 2

Distribution of Formulaic Sequences (FLS) Data Across the Two Time Intervals

\begin{tabular}{lcccccc}
\hline & $\begin{array}{c}\text { FLS/Min. } \\
\text { Time 1 }\end{array}$ & $\begin{array}{c}\text { FLS/Min. } \\
\text { Time 2 }\end{array}$ & $\begin{array}{c}\text { Words/FLS } \\
\text { Time 1 }\end{array}$ & $\begin{array}{c}\text { Words/FLS } \\
\text { Time 2 }\end{array}$ & $\begin{array}{c}\text { FLS \% } \\
\text { Time 1 }\end{array}$ & $\begin{array}{c}\text { FLS \% } \\
\text { Time 2 }\end{array}$ \\
\hline Task 1 & 20.00 & 13.47 & 2.42 & 2.11 & 41 & 32 \\
Task 2 & 23.07 & 12.67 & 2.32 & 2.18 & 45 & 32 \\
Task 3 & 23.53 & 15.40 & 2.47 & 2.27 & 43 & 33 \\
\hline Total & 22.20 & 13.84 & 2.40 & 2.19 & 43 & 32 \\
\hline
\end{tabular}



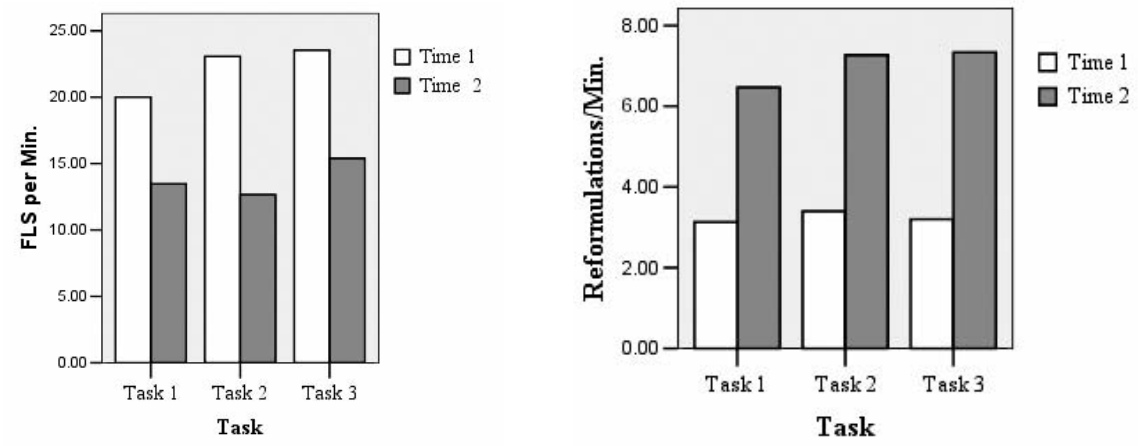

Figure 1. Effect of time on both analyzability and accessibility measures.

The participants seemed to fall back on the analytic processing system when time pressure peaked. This behavioral course is exemplified by the increased instances of pausing and reformulations, which are indicative of explicit use of language and high degrees of control. As shown in Figure 1, scores in one processing mode were reversed in the other. This reflects the negative correlations reported in Table 1. Overall, analysis of these results suggests an on-off relationship between the two processing modes, thus confirming Hypothesis One. That is, where L2 speakers are given the opportunity to plan their performance ahead, they take advantage of their memory-based knowledge. Conversely, when their capacity is stretched by mounting time pressure, they resort to activating their rule-based knowledge.

It was also hypothesized that the distribution of FLS could account for the trade-off relationship between oral performance areas. An analysis of variance was performed to examine the extent of the effect of time pressure, which is strongly associated with FLS distribution, compared with the effect of the experimental tasks on oral performance. In this respect, results from multivariate $F$ tests of Wilks' Lambda were largely significant. The dependent variables of fluency, complexity, and accuracy were significantly influenced by Time $(F(3,172)=259.62, p<.05)$, Task $(F(6,344)=13.49, p<.05)$, and Time-by-Task interaction effects $(F(6,334)=2.33, p<.05)$. These MANOVA results are consistent with the follow-up ANOVA results reported in Table 3. Both analyses of variance robustly confirm the significant effect of the Time variable (e.g., Fluency: $F(1,88)=302.83, p<.001$, partial $\left.\eta^{2}=.64\right)$. The Task variable effect, however, shows less significance for the three performance variables. Despite the high statistical significance across the three performance areas, commensurate effect size estimates suggest low significance levels (e.g., Complexity: $F(2,87)=4.42, p=.01$, partial $\left.\eta^{2}=.05\right)$. As to Time-by-Task effect, results are not statistically significant. 
Table 3

Results From the ANOVAs on Time and Task Effects on Oral Performance

\begin{tabular}{lccccccc}
\hline \multirow{2}{*}{ Source } & $\begin{array}{c}\text { Dependent } \\
\text { Variable }\end{array}$ & $\begin{array}{c}\text { Sum of } \\
\text { Squares }\end{array}$ & Df & Mean Square & $F$ & $p$ & $\begin{array}{c}\text { Partial } \\
\eta^{2}\end{array}$ \\
\hline \multirow{2}{*}{ Time } & Fluency & 34.77 & 1 & 34.77 & 302.83 & .00 & .64 \\
& Complexity & 4.10 & 1 & 4.10 & 134.51 & .00 & .44 \\
& Accuracy & 15.45 & 1 & 15.45 & 449.88 & .00 & .72 \\
\hline \multirow{2}{*}{ Task } & Fluency & 7.94 & 2 & 3.97 & 34.56 & .00 & .28 \\
& Complexity & .27 & 2 & .13 & 4.42 & .01 & .05 \\
& Accuracy & .32 & 2 & .16 & 4.71 & .01 & .05 \\
\hline \multirow{2}{*}{ Time * Task } & Fluency & .45 & 2 & .22 & 1.94 & .15 & .02 \\
& Complexity & .10 & 2 & .05 & 1.64 & .20 & .02 \\
& Accuracy & .23 & 2 & .11 & 3.32 & .04 & .04 \\
\hline
\end{tabular}

Note. The mean difference is significant at the .05 level.

Based on the results shown in Table 1, it follows that FLS use is at its highest when time pressure is low (Time 1) and dwindles when time pressure peaks (Time 2). In this respect, this study sought to test whether formulaicity, an indicator of the holistic-processing mode that competes with the analyticprocessing mode, underlies a surface competition at the level of the three performance areas of fluency, complexity, and accuracy. The Pearson's correlation results displayed in Table 4 indicate significant correlation coefficients between the three FLS dimensions (frequency, density, and percentage of use) and the three performance areas. The strongest of the correlations re-

Table 4

Correlational Results for FLS and Oral Performance

\begin{tabular}{|c|c|c|c|c|c|c|}
\hline & 1 & 2 & 3 & 4 & 5 & 6 \\
\hline 1. FLS/Min. & - & $.33^{*}$ & $.79^{*}$ & $.80^{*}$ & $-.46^{*}$ & $.72^{*}$ \\
\hline 2. Words/FLS & & - & $.45^{\star}$ & $.43^{*}$ & $-.33^{*}$ & $.29^{*}$ \\
\hline 3. FLS \% & & & - & $.51^{*}$ & $-.45^{\star}$ & $.62^{*}$ \\
\hline 4. Fluency & & & & - & $-.46^{*}$ & $.69^{*}$ \\
\hline 5. Complexity & & & & & - & $-.60^{*}$ \\
\hline 6. Accuracy & & & & & & - \\
\hline
\end{tabular}


volves around FLS frequency (Fluency: $r=.80$; Complexity: $r=-.46$; Accuracy: $r=.72$ ). Formulaicity is positively correlated with fluency and accuracy, yet negatively associated with complexity, thus implying the trade-off concept and confirming Hypothesis Two. This could be because formulaic sequences are fixed to some degree and thus delivered more fluently and with unanalyzed accuracy. In other words, the speakers' reliance on formulaic language enabled them to attend to fluency and accuracy and not complexity. However, when they were under-resourced due to time pressure, they traded off fluency and accuracy for complexity.

\section{Discussion}

In this study I aimed to chart the nature of the relationship between the holistic-processing mode and the analytic-processing mode during L2 speakers' task engagement. Results confirmed the claim that L2 learners use the holistic-processing mode in least demanding task conditions and fall back on the analytical-processing mode when their working memory is overburdened as time pressure increases. This finding qualifies as an empirical validation of Widdowson's (1989) assertion that analyzability overrides accessibility when task difficulty intensifies. In this vein, Widdowson presumes that "When the association of lexis and context became insufficient to establish meaning, grammatical rules would be called in to assist, to adapt and adjust the lexis with whatever syntactic fittings were required" (p. 136). Also, this study replicates to some extent Foster's (2001) findings that the size of L2 learners' lexical repertoires interacts with the pre-task planning variable in its effect on oral performance. More precisely, it is the availability of formulaic resources that determines one's mode of processing.

Foster's study (2001) is perhaps the only one in the processing-based research line that empirically accounts for the limited-capacity concept with reference to the two processing modes. The idea of rivalry between the two modes, although not recent (Skehan, 1998), does not extend beyond the level of theorizing, which is perhaps explained by the methodological limitations underlying the study of formulaic language. In her endeavor to revisit Foster and Skehan (1996), Foster (2001) refers to the problems of the psychometric properties of reliability and validity related to the technicalities of FLS coding. Notwithstanding this methodological caveat, shedding empirical light on the FLS in the information-processing framework seems to be to be rewarding in the sense that it refines our understanding of attentional capacity being merely constrained by psycholinguistic demands at the micro-levels of processing (Levelt, 1989) or by the pedagogically based competition between fluency, complexity, and accuracy (Skehan, 1998).

The results of this study support Foster's (2001) assertion that it is the availability of and access to formulaic language that lie behind the choice of one processing mode over the other. Accordingly, the trade-off relationship 
between performance areas is only a surface reflection of how capacitystretched L2 learners exploit their formulaic repertoire. On the one hand, when given the opportunity to plan their performance, the speaker participants drew on prefabricated word combinations that would leave them sufficient attentional resources for monitoring their output, and so fluency and accuracy were improved. Complexity, however, was less important at this point because of the total focus on the formulaic constructions, which bear some ready-made syntactic properties, hence allowing little syntactic animation in speech. On the other hand, when pressed for time and thus unable to retrieve formulaic sequences automatically from the memory-based system, the under-resourced informants had to process output analytically, with the result that complexity improved as a function of the focus on the rule-based system to the detriment of fluency and accuracy.

The size and quality of one's exemplar-based system decidedly determines one's processing behavior. These results seem to agree with Foster (2001) and Temple (2000) in their assertions that NSs and NNSs engage in varying processing patterns because of the differences in terms of their formulaic repertoires and speaking goals. As far as native speakers are concerned, they have a larger and more sophisticated range of formulaic language, and they distinguish grammatical regularities from these chunks. Much of their speech is not rule-based, although they tend to use their analytic-processing mode when tasks are familiar and undemanding to be creative, because conventionalized formulaic language does not meet the originality requirements of novel ideas. Their use of the holistic-processing mode is, however, associated with unfamiliar tasks where formulaic language serves as a communication strategy to maintain continual yet less complex speech. These FLS instances (e.g., discourse fillers) may have the organizational value of giving learners the opportunity for on-line planning (Yuan \& Ellis, 2003) in real-time performance.

The limited formulaic depository of NNSs and their form-focused speaking goals prompted them toward the reverse processing pattern: using an exemplar mode in an undemanding task with little analyzability to the advantage of fluency and accuracy. This perhaps explains why the informants in this study scored highest on accuracy at the level of the pressure-free Time 1; their accuracy was positively associated with the use of FLS (Table 4). Surely the effortless access to FLSs at Time 1 would spare them inaccurate performance because of their minimal use of grammatical rules. Conversely, their recourse to the rule-based system was perhaps driven by their inability easily to retrieve sufficient FLSs to meet the pressing communication needs. They made greater use of pausing and reformulations as time-gaining strategies to compensate for the decline in FLS use (Figure 1). In fact, these capacity-stretched learners seemed to experience the problem that their encoding system was unable to match the complex verbal plans from the conceptual- 
izer with appropriate prefabricated morphosyntactic and lexical combinations, because much of this knowledge might not be fully proceduralized. Their long pauses to catch up on the delayed lexical retrieval (Temple, 2000) and frequent reformulations helped them try novel message-form mappings that required more morphosyntactic elaboration, as evidenced in the increase of complexity.

The research significance of these results consists in revisiting the longestablished account of limited-capacity concept. Accordingly, it appears too simplistic categorically to attribute attentional trade-offs to the competition between the three areas of performance, whereas these pedagogically conceived areas are in turn mobilized by two competing processing modes. For an account of trade-offs (e.g., between complexity and fluency as in this study and in Foster, 2001) to be tenable, it needs to consider the extent of development of L2 speakers' knowledge system in terms of formulaic storage and automaticity of rules. As in the above-mentioned difference between NNSs and NSs, one's range of formulaic language, together with variation related to task types and sequencing conditions, may suggest a more concise and fine-grained account of the capacity concept and task difficulty. In fact, researching formulaicity alongside task effect may well offset the deterministic frame of mind in the processing-based approach that according to Ellis (2000), "assumes that there are properties in a task that will predispose, even induce, learners to engage in certain types of language use" (p. 197).

These results have direct implications for practitioners interested particularly in developing language learners' speaking abilities. Although no experimental attempt was made to expose the informants of the present study to formulaic input, analysis of the results revealed that much of the variance in their output was closely associated with the scope of formulaicity in their performance. Indeed, raising teachers' awareness of this type of variation may advance their view and treatment of task sequencing. Accordingly, their understanding of difficulty, being related not only to speaking task features or conditions, but also to learners' depository of formulaic language, may encourage them to feature formulaicity in their teaching agendas. This may be beneficial mostly to novice L2 learners whose fledgling interlanguage system would draw on formulaic sequences to improve the maintenance and control in their flow of communication. The processing advantage provided by formulaicity may, therefore, present a source of motivation to struggle toward task completion and reduce the anxiety of communication breakdowns.

The study demonstrates that the competition between fluency and complexity is only a surface manifestation of two processing modes that underlie the two developmental aspects of automatization and restructuring. Practitioners' awareness of the nature of the relationship between these two ends may usefully inform pedagogical decisions so as to ensure a balanced consideration of automatization and restructuring. It is hence necessary that L2 
teachers provide the contexts and the pedagogical means to promote formulaicity, especially among advanced L2 learners, because acquiring a rich and varied lexicalized language will enable the latter to reach native-like status in how language is processed and used. However, restructuring is equally recommended, as these learners need to reflect on their formulaic repertoire and develop more syntactically complex language. Drawing L2 learners' attention to restructuring their formulaic and unanalyzed language would minimize the risk of early fossilization because as Foster (2001) states, "regularly used examples [of FLS] will become too deeply entrenched in memory to be dislodged easily by further reflection" (p. 90).

\section{Conclusion}

This study examined how L2 learners employ their dual-mode system in performing narrative tasks. Analysis of the findings suggests a trade-off relationship between fluency and complexity, which reflects competition between the holistic-processing mode and the analytic-processing mode. Under time pressure, the informants exhausted their formulaic repertoire and ultimately switched to a more syntactically convoluted performance punctuated by a large number of pauses and reformulations. Contrary to the assumptions of earlier L2 processing-based research that L2 learners' limited-capacity is mobilized by task difficulty, it follows that the quality of L2 learners' formulaic language is equally influential in defining the nature of the fluency-complexity trade-off. It also needs to be pointed out that accuracy benefits in part from formulaicity, because the use of the latter as a time-buying strategy might help the participants to apportion extra time to monitor their output in the context of instructed L2 learning.

Even with the effort in this study to underscore the role of formulaicity in oral production processes, the fundamental problem continues to be the coding procedure implemented here. It may not be completely valid and reliable because of the small number of rater participants. Added to this, the data were pooled from a small corpus, although it did have the advantage of being recorded from speakers who shared almost the same linguistic background, age, proficiency level, and were performing the same task under similar experimental conditions. It remains unclear, however, whether the results are generalizable to all learners at beginning or advanced levels given that the size of FLS repertoire differs according to learners' L2 learning experience. Therefore, in order to enhance the generalizability of the findings, follow-up research with larger samples under various experimental conditions is needed. It would also be worth investigating whether varying attainment levels share the same processing engagement patterns and whether the longitudinal picture of such patterns might further our understanding of the nature of interlanguage development. 


\section{Notes}

${ }^{1}$ It is widely reported in task-based research that varied task types and task conditions trigger varying effects on oral performance, and so it is difficult to come to a standard conclusion about the nature of the trade-offs (i.e., which factor prioritizes a given language area over the other(s)). ${ }^{2}$ Time-pressure operationalization was first informed by results from a similar study (Ben Maad, 2008) in which speech performances showed more variation as the participants progressed in task engagement. The choice of 60 seconds as the cutoff time may not mark the exact beginning of time pressure effect, but it would have the technical advantage of comparing data sets usefully.

\section{Acknowledgments}

I am grateful to Pauline Foster, Habib Abdesslem, and two anonymous reviewers for their invaluable comments on an earlier draft of this article. I am also indebted to the students who participated in the study and to those who assisted me in this work.

\section{The Author}

Mohamed Ridha Ben Maad is an assistant professor currently teaching applied English in the Higher Institute for Childhood Education, Carthage-Tunis, Tunisia. His main research interests are in applied linguistics and second-language acquisition. He also participates in research projects related to early childhood education.

\section{References}

Bachman, L.F. (1990). Fundamental considerations in language testing. Oxford, UK: Oxford University Press.

Ben Maad, M.R. (2008). Time pressure and within-task variation in EFL oral performance. Electronic Journal of Foreign Language Teaching, 5, 5-12.

Ellis, R. (2000). Task-based research and language pedagogy. Language Teaching Research, 4 , 193-220.

Fletcher, M. (1997). Picture stories please. Hythe, UK: Brain Friendly Publications.

Foster, P. (2001). Rules and routines: A consideration of their role in the task-based language production of native and non-native speakers. In M. Bygate, P. Skehan, \& M. Swain (Eds.), Researching pedagogic tasks: Second language learning, teaching and testing (pp. 75-93). Harlow, UK: Longman.

Foster, P., \& Skehan, P. (1996). The influence of planning and task type on second language performance. Studies in Second Language Acquisition, 18, 299-323.

Levelt, W.J.M. (1989). Speaking: From intention to articulation. Cambridge, MA: MIT Press.

Pawley, A., \& Syder, F. (1983). Two puzzles for linguistic theory: Nativelike selection and nativelike fluency. In J. Richards \& R. Schmidt (Eds.), Language and communication (pp. 191268). London: Longman.

Read, J., \& Nation, P. (2004). Measurement of formulaic sequences. In N. Schmitt (Ed.), Formulaic sequences: Acquisition, processing and use (pp. 23-35). Amsterdam: John Benjamins.

Sinclair, J. (1991). Corpus, concordance, collocation. Oxford, UK: Oxford University Press.

Skehan, P. (1998). A cognitive approach to language learning. Oxford, UK: Oxford University Press.

Tabachnick, B.G., \& Fidell, L.S. (2001). Using multivariate statistics (4th ed.). New York: Allyn and Bacon.

Tavakoli, P., \& Skehan, P. (2005). Strategic planning, task structure, and performance testing. In R. Ellis (Ed.), Planning and task performance in a second language (pp. 239-273). Amsterdam: John Benjamins.

Temple, L. (2000). Second language learner speech production. Studia Linguistica, 54, 288-297.

Weinert, R. (1995). The role of formulaic language in second language acquisition: A review. Applied Linguistics, 16, 180-205. 
Widdowson, H.G. (1989). Knowledge of language and ability for use. Applied Linguistics, 10, 128-137.

Wolfe-Quintero, K., Inagaki, S., \& Kim, H.-Y. (1998). Second language development in writing: Measures of fluency, accuracy and complexity. (Technical report 17). Manoa, HI: University of Hawai'i Press.

Yuan, F., \& Ellis, R. (2003). The effects of pre-task planning and on-line planning on fluency, complexity and accuracy in L2 monologic oral production. Applied Linguistics, 24, 1-27. 